\title{
State Representations of Linear Systems with Output Constraints*
}

\begin{abstract}
J. M. Schumacher $\dagger$
Abstract. We derive state space representations for linear systems that are described by input/state/output equations and that are subjected to a number of constant linear constraints on the outputs. In the case of a general linear system, the state representation of the constrained system is shown to be essentially nonunique. For linear Hamiltonian systems satisfying a nondegeneracy condition, there is a natural and unique choice of the representation which preserves the Hamiltonian structure. In the linear systems setting we give an algebraic proof that a system with $n$ degrees of freedom under $k$ constraints becomes a system with $n-k$ degree of freedom. Similar results are obtained for linear gradient systems.
\end{abstract}

Key words. Constrained linear system, Gradient systems, Hamiltonian systems, State representation.

\section{Introduction and Preliminaries}

The purpose of this paper is to contribute to the understanding of the relation between realization theory and physical modeling. Here, we understand "realization theory" in a broad sense, as the theory of equivalent system representations. "Physical modeling" is understood as the construction of dynamical models for physical systems using constitutive equations and element connections.

Methods for physical modeling, in the above sense, are basic ingredients in every engineering curriculum. Although this is not always made very explicit, the central issue addressed by these techniques is the transformation of a given system of differential and algebraic relations to another, more suitable, form. For instance, we may obtain a system description for an electrical network by writing down the equations that follow from the laws of Kirchhoff, Ohm, and Faraday. The resulting system of equations will in general involve algebraic as well as differential relations. The modeling problem is to obtain an equivalent description in standardized (for instance, input/state/output) form. The setting up of Lagrangian equations for constrained mechanical systems follows essentially the same route.

The problem of transforming a system of algebraic and differential equations to a standardized form has also been considered in system theory, for instance, by

* Date received: April 18, 1988. Date revised: September 15, 1988.

† Centre for Mathematics and Computer Science, P.O. Box 4079, 1009 AB Amsterdam, The Netherlands. 
Rosenbrock [R2] and Luenberger [L2], and more recently by Willems [W2] and the author [S4]. However, it appears that these methods, which were developed for the class of general linear systems, are not able to bring out certain properties than we expect to see in the context of physical modeling. A simple example may help to clarify this point. Consider two masses attached to springs, each subject to an external force, and assume that the motion is constrained by a rigid connection between the two masses. Equations may be written down as follows:

$$
\begin{aligned}
m_{1} \ddot{y}_{1}(t)+k_{1} y_{1}(t) & =u_{1}(t), \\
m_{2} \ddot{y}_{2}(t)+k_{2} y_{2}(t) & =u_{2}(t), \\
y_{1}(t) & =y_{2}(t) .
\end{aligned}
$$

In the framework of Willems [W2], the "behavior" defined by these equations is simply the set of all trajectories $\left(y_{1}(\cdot), y_{2}(\cdot), u_{1}(\cdot), u_{2}(\cdot)\right)^{\mathrm{T}}$ that satisfy (1.1)-(1.3). Among these trajectories, there are harmonic solutions, for instance,

$$
\begin{aligned}
& y_{1}(t)=y_{2}(t)=\sin \omega t, \\
& u_{1}(t)=\left(k_{1}-m_{1} \omega^{2}\right) \sin \omega t, \\
& u_{2}(t)=\left(k_{2}-m_{2} \omega^{2}\right) \sin \omega t .
\end{aligned}
$$

These solutions show no value of $\omega$ having a special significance. The situation changes, however, if we associate with each trajectory the function

$$
W(t)=u_{1}(t) \dot{y}_{1}(t)+u_{2}(t) \dot{y}_{2}(t)
$$

which expresses the work done on the system. For the harmonic solutions above, we get

$$
W_{h}(t)=\left[\left(k_{1}+k_{2}\right)-\left(m_{1}+m_{2}\right) \omega^{2}\right] \omega \sin \omega t \cos \omega t .
$$

From this, we see that there is one particular value of $\omega$ that leads to a nontrivial harmonic solution in which no work is done on the system:

$$
\omega_{\mathrm{n}}=\left(\frac{k_{1}+k_{2}}{m_{1}+m_{2}}\right)^{1 / 2} .
$$

Of course, $\omega_{\mathrm{n}}$ is, according to common terminology, the natural frequency of the connected system.

Our example is sufficiently general to warrant the conclusion that the natural frequencies of a connected system will in general not be recognized if we follow the viewpoint of [W2]. This is no surprise since the framework of [W2] is developed for the class of general linear systems, and, at this level, there is no notion of energy which serves to distinguish the "natural" frequencies from other frequencies. The same phenomenon occurs in any other setting for transformations of general systems of linear equations, such as the one provided by [R2].

The present paper aims to explain the seeming discrepancy between modeling with the techniques of [W2] and [S4] on the one hand, and "physical modeling" on the other hand. We shall show that a link between the two can be formulated in 
geometric terms, i.e., using particular structures of vector spaces, in the spirit of modern treatments of classical mechanics (see [AM]). Since our primary purpose is to establish the existence of this link, full generality will not be pursued; in particular, the treatment here is restricted to linear systems, and, even more in particular, to systems of algebraic and differential equations that can be written as standard input/state/output systems together with a number of static constraints on the outputs. Furthermore, we feel free to use additional assumptions (although not unreasonable ones) when this is convenient. Although we concentrate on the connection between the general linear case and the more specific cases of Hamiltonian linear systems and gradient linear systems, we can reasonably expect that the links established in the linear situation can be extended to the nonlinear case (see [S2] and [S3]).

We now quickly review a number of definitions and results from linear algebra and from linear system theory that will be needed below. Consider first a linear system in input/state/output representation

$$
\begin{aligned}
& \dot{x}(t)=A x(t)+B u(t), \\
& y(t)=C x(t)+D u(t),
\end{aligned}
$$

where $x(t), u(t)$, and $y(t)$ take values in finite-dimensional linear spaces $X, U$, and $Y$, and where $A, B, C$, and $D$ are linear mappings between the appropriate spaces. To alleviate the notational burden, the time argument is often suppressed below. With the system (1.10)-(1.11), we associate the transfer matrix

$$
G(s)=C(s I-A)^{-1} B+D .
$$

The transfer matrix $G(s)$ can be considered as a matrix over the field $\mathbb{R}(s)$ of rational functions with real coefficients. The system (1.10)-(1.11) is said to be invertible if $G(s)$ is invertible as a matrix over $\mathbb{R}(s)$; similar definitions are used for left and right invertibility. Because $G(s)$ as defined by (1.12) is regular at infinity, we can also consider $G(s)$ as a matrix over the ring $\mathbb{R}_{\infty}(s)$ of proper rational functions with real coefficients. The ring $\mathbb{R}_{\infty}(s)$ is a principal ideal domain with a unique maximal ideal generated by the function $s^{-1}$; we can therefore obtain a Smith normal form of $G(s)$ (see, for instance, p. 42 of [M1]) in which all nonzero elements are of the form $s^{-k_{j}}$, $k_{j} \geq 0$ (see [H1]). The indices $k_{j}$ are called the orders of the zeros at infinity of $G(s)$.

The orders of the zeros at infinity can also be expressed more directly in terms of the mappings $A, B, C$, and $D$. For this, we need the " $V^{*}$-algorithm" (given on p. 91 of [W3] for the case $D=0$, and in [A1] for the more general case in which $D$ may be nonzero). Given $A, B, C$, and $D$ as mappings between the state space $X$, the output space $Y$, and the input space $U$, define

$$
\begin{aligned}
V^{0} & =X, \\
V^{k+1} & =\left\{x \in V^{k} \mid \exists u \in U \text { such that } A x+B u \in V^{k} \text { and } C x+D u=0\right\} .
\end{aligned}
$$

This defines a decreasing sequence of subspaces of $X$. Because $\operatorname{dim} X$ is finite, there must be some value of $k$ for which $V^{k+1}$ equals $V^{k}$, and then $V^{k+j}$ will be equal to $V^{k}$ for all $j \geq 0$. This limit subspace is denoted by $V^{*}(A, B, C, D)$ or simply by $V^{*}$ if the reference is clear. Now, it has been shown in [M2] (see also [NS]) that the 
number of zeros at infinity of order $\geq k$ of the system (1.10)-(1.11) is, for $k \geq 1$, equal to

$$
p_{k}=\operatorname{dim}\left(V^{k-1} \cap B[\operatorname{ker} D]\right)-\operatorname{dim}\left(V^{*} \cap B[\operatorname{ker} D]\right) .
$$

When $D=0$, there are no zeros at infinity of order zero so that $p_{1}$ is equal to the rank of the transfer matrix:

$$
\text { rank } G(s)=\operatorname{dim} \operatorname{im} B-\operatorname{dim}\left(\operatorname{im} B \cap V^{*}\right) .
$$

This formula was first proved, by a different method, in $[\mathrm{CM}]$. For the " $D \neq 0$ " case, we have

$$
\operatorname{dim} \operatorname{ker} G(s)=\operatorname{dim}\left\{u \in U \mid B u \in V^{*} \text { and } D u=0\right\} .
$$

The subspace $V^{*}$ is the maximal subspace $V$ of $X$ having the property that there exists a feedback mapping $F: X \rightarrow U$ such that

$$
(A+B F) V \subset V
$$

and

$$
V \subset \operatorname{ker}(C+D F)
$$

(see [W3] and [A1]).

The system (1.10)-(1.11) is said to have uniformly kth order zeros at infinity if it is invertible and all its zeros at infinity are of order $k$. If $k=0$, this simply means that $D$ must be invertible. For larger values of $k$, it is easily verified that (1.10)-(1.11) has uniformly $k$ th-order zeros at infinity if and only if $D=0, C A^{j} B=0$ for $j=0$, $\ldots, k-2$, and $C A^{k-1} B$ is invertible. Also, in this case, it is seen from the defining algorithm (1.13)-(1.14) that

$$
V^{*}=V^{k}=\operatorname{ker} C \cap \operatorname{ker} C A \cap \cdots \cap \operatorname{ker} C A^{k-1} .
$$

Now, let us recall some definitions from linear algebra that will be needed below (see, for instance, Chapter XIII of [L1]). Let $X$ be a vector space over a field $K$ of characteristic $\neq 2$. A symmetric form on $X$ is a bilinear mapping $f: X \times X \rightarrow K$ that satisfies $f\left(x_{1}, x_{2}\right)=f\left(x_{2}, x_{1}\right)$ for all $x_{1}$ and $x_{2}$ in $X$. The form is said to be nondegenerate if $f\left(x_{1}, x_{2}\right)=0$ for all $x_{2}$ in $X$ only if $x_{1}=0$. We use square brackets below as a notation for symmetric forms, so we write $\left[x_{1}, x_{2}\right]$ rather than $f\left(x_{1}, x_{2}\right)$. Note that positively is not required in the definition of a symmetric form. A linear mapping $A: X \rightarrow X$ is said to be symmetric with respect to $[\cdot, \cdot]$ if

$$
\left[A x_{1}, x_{2}\right]=\left[A x_{2}, x_{1}\right]
$$

for all $x_{1}$ and $x_{2}$ in $X$.

A bilinear mapping $g: X \times X \rightarrow K$ is said to be an alternating form if $g\left(x_{1}, x_{2}\right)=$ $-g\left(x_{2}, x_{1}\right)$ for all $x_{1}$ and $x_{2}$ in $X$. The form is said to be nondegenerate under the same condition as in the symmetric case. We use round brackets to denote alternating forms, writing $\left(x_{1}, x_{2}\right)$ rather than $g\left(x_{1}, x_{2}\right)$. A vector space equipped with a nondegenerate alternating form is called a symplectic space; such spaces are always even-dimensional [L1, p. 371]. For a symplectic space, we can always find a symplectic basis, i.e., a basis in which the alternating form $\left(x_{1}, x_{2}\right)$ can be written as 
$x_{1}^{\mathrm{T}} J x_{2}$ with

$$
J=\left(\begin{array}{cc}
0 & -I \\
J & 0
\end{array}\right) .
$$

Given a subspace $V$ of a symplectic space $X$, its symplectic orthoplement is

$$
V^{(\perp)}=\{x \in X \mid(v, x)=0 \text { for all } v \in V\} .
$$

Simple rules such as $\operatorname{dim} V^{(\perp)}=\operatorname{codim} V$ and $(V \cap W)^{(\perp)}=V^{(\perp)}+W^{(\perp)}$ are freely used.

Now, let $U$ and $Y$ be vector spaces over a field $K$. A bilinear mapping $h: U \times Y \rightarrow K$ is called a duality between $U$ and $Y$ if it is nondegenerate in the sense that $h(u, y)=0$ for all $u \in U$ implies $y=0$, and $h(u, y)=0$ for all $y \in Y$ implies $u=0$. Dualities are denoted below by sharp brackets: we write $\langle u, y\rangle$ rather than $h(x, y)$. Spaces that are connected by a duality must have equal dimension. If $Y_{1}$ is a subspace of $Y$, its orthogonal space is the subspace of $U$ that is defined by

$$
Y_{1}^{\perp}=\left\{u \in U \mid\langle u, y\rangle=0 \text { for all } y \in Y_{1}\right\} .
$$

Again, simple rules concerning dimensions and concerning sums and intersections are used without comment.

If $X$ is a space equipped with a symmetric form, then every subspace of $X$ can also be equipped with a symmetric form in a natural way; we simply take the restriction of the form to the given subspace. The same is not true for symplectic spaces, as is already evident from the fact that symplectic spaces must have even dimension. However, we do have the following result.

Lemma 1.1. Let $X$ be a symplectic space and let $V$ be a subspace of $X$. Let $W$ be a complement of $V \cap V^{(\perp)}$ in $V$. Under these conditions, $W$ is a symplectic space with respect to the restriction of the alternating form on $X$ to $W$.

Proof. There is a natural isomorphism between $W$ and the factor space $V /\left(V \cap V^{(\perp)}\right)$, and this isomorphism makes the restricted alternating form on $W$ correspond to the induced alternating form on the factor space. It is easily seen that $V /\left(V \cap V^{(\perp)}\right)$ is symplectic with respect to the induced form.

In a similar fashion, we can prove that if $U$ and $Y$ are dual spaces and $Y_{1}$ is a subspace of $Y$, then there is a natural induced duality between $Y_{1}$ and any complement of $Y_{1}^{\perp}$ in $U$.

\section{The General Linear System Case}

Consider a linear system in input/state/output form:

$$
\begin{aligned}
& \dot{x}(t)=A x(t)+B u(t), \\
& y(t)=C x(t)+D u(t) .
\end{aligned}
$$

Now assume that we constrain the outputs to lie in a certain subspace of the output 
space $Y$ :

$$
y(t) \in Y_{1}, \quad Y_{1} \subset Y .
$$

The three equations (2.1)-(2.3) still describe a linear system in the sense of [W1] (the set of input/output functions $(u(\cdot), y(\cdot))$ for which there exists a state function $x(\cdot)$ such that $(2.1),(2.2)$, and $(2.3)$ are satisfied forms a linear subspace of the vector space of all input/output functions), but it is, of course, not a description in state space form. So we may ask how to obtain minimal state representations for the system described by (2.1)-(2.3). We have to specify a notion of equivalence of course; we use external equivalence (see [W1], [BY] and [S4]). As a matter of fact, the question just posed is a particular instance of a problem for which a solution algorithm was given in [S4]. So we only have to see what the procedure in that paper leads to for the special case at hand.

Let $H$ be a linear mapping acting on $Y$ such that ker $H=Y_{1}$. Let $V^{*}\left(Y_{1}\right)$ denote the subspace $V^{*}(A, B, H C, H D)$ of $X$. In other words, $V^{*}\left(Y_{1}\right)$ is the limit of the sequence of subspaces defined by

$$
\begin{aligned}
V^{0} & =X, \\
V^{k+1} & =\left\{x \in V^{k} \mid \exists u \in U \text { such that } A x+B u \in V^{k} \text { and } C x+D u \in Y_{1}\right\} .
\end{aligned}
$$

Decompose $X$ as $X=X_{1} \oplus X_{2}$ where $X_{1}=V^{*}\left(Y_{1}\right)$. Let the mapping $F: X \rightarrow U$ be such that $V^{*}\left(Y_{1}\right)$ is $(A+B F)$-invariant and $(C+D F) V^{*}\left(Y_{1}\right) \subset Y_{1}$; we can always arrange that ker $F$ contains $X_{2}$ (this simplifies the notation somewhat, but is otherwise inessential). Equations (2.1) and (2.2) may now be rewritten in the form

$$
\begin{aligned}
& \dot{x}_{1}=\left(A_{11}+B_{1} F_{1}\right) x_{1}+A_{12} x_{2}+B_{1}\left(u-F_{1} x_{1}\right), \\
& \dot{x}_{2}=A_{22} x_{2}+B_{2}\left(u-F_{1} x_{1}\right), \\
& y=\left(C_{1}+D F_{1}\right) x_{1}+C_{2} x_{2}+D\left(u-F_{1} x_{1}\right) .
\end{aligned}
$$

By construction, we have $H\left(C_{1}+D F_{1}\right)=0$, so that the restriction (2.3) can be written as

$$
H C_{2} x_{2}+H D\left(u-F_{1} x_{1}\right)=0 .
$$

We temporarily introduce new inputs by defining

$$
u-F_{1} x_{1}=\left[\begin{array}{ll}
G_{1} & G_{2}
\end{array}\right]\left(\begin{array}{l}
v_{1} \\
v_{2}
\end{array}\right),
$$

where $G=\left[\begin{array}{ll}G_{1} & G_{2}\end{array}\right]$ is an invertible mapping satisfying

$$
\operatorname{im} G_{1}=\left\{u \in U \mid B u \in V^{*}\left(Y_{1}\right) \text { and } D u \in Y_{1}\right\} \text {. }
$$

This gives us the equations $B_{2} G_{1}=0$ and $H D G_{1}=0$. We can now rewrite (2.7) as

$$
\dot{x}_{2}=A_{22} x_{2}+B_{2} G_{2} v_{2} \text {, }
$$

whereas the constraints can be formulated as

$$
H C_{2} x_{2}+H D G_{2} v_{2}=0 \text {. }
$$

It is shown in [S4] that the only solution of the set of differential and algebraic 
equations given by (2.12) and (2.13) is the zero solution $x_{2}(t)=0, v_{2}(t)=0$. So, the input/output trajectories of the system (2.1)-(2.2) that satisfy the constraint (2.3) are described by

$$
\begin{aligned}
\dot{x}_{1} & =\left(A_{11}+B_{1} F_{1}\right) x_{1}+B_{1} G_{1} v_{1}, \\
y & =\left(C_{1}+D F_{1}\right) x_{1}+D G_{1} v_{1}, \\
u & =G_{1} v_{1}+F_{1} x_{1} .
\end{aligned}
$$

Now, we want to eliminate the auxiliary input $v_{1}$ in order to arrive at a description in standard state space form. This can be done from (2.16) since $G_{1}$ is injective. Write

$$
\left[\begin{array}{ll}
G_{1} & G_{2}
\end{array}\right]^{-1}=\left(\begin{array}{l}
K_{1} \\
K_{2}
\end{array}\right)
$$

so that $v_{1}=K_{1} u-K_{1} F_{1} x_{1}$. Equations (2.14) and (2.15) can be written as

$$
\begin{aligned}
\dot{x}_{1} & =\left(A_{11}+B_{1}\left(I-G_{1} K_{1}\right) F_{1}\right) x_{1}+B_{1} G_{1} K_{1} u, \\
y & =\left(C_{1}+D\left(I-G_{1} K_{1}\right) F_{1}\right) x_{1}+D G_{1} K_{1} u, \\
K_{2} u & =K_{2} F_{1} x_{1} .
\end{aligned}
$$

This is the state space form derived in [S4]. Note that the original inputs $u(t)$ have been split up into certain combinations $K_{1} u(t)$ which still function as inputs, and other combinations $K_{2} u(t)$ whose values are determined by the constraints. The latter variables are, therefore, described as outputs.

The state space equations above can be rewritten to give them a slightly nicer appearance. By construction, we have $K_{1} G_{1}=I$ so that $I-G_{1} K_{1}$ is a projection with kernel

$$
\operatorname{ker}\left(I-G_{1} K_{1}\right)=\operatorname{im} G_{1}=\left\{u \in U \mid B u \in V^{*}\left(Y_{1}\right) \text { and } D u \in Y_{1}\right\} .
$$

The image of the projection is $\operatorname{im} G_{2}$, which may be any complement in im $G_{1}$ in $U$. Note that if $F: X \rightarrow U$ is any mapping which acts on $X_{1}$ like $\left(I-G_{1} K_{1}\right) F_{1}$, then $F$ has the properties

$$
(A+B F) V^{*}\left(Y_{1}\right) \subset V^{*}\left(Y_{1}\right)
$$

and

$$
(C+D F) V^{*}\left(Y_{1}\right) \subset Y_{1},
$$

and satisfies $\operatorname{im} F \subset \operatorname{im~} G_{2}$; and conversely, every $F$ that fulfills these requirements agrees on $X_{1}$ with some mapping of the form $\left(I-G_{1} K_{1}\right) F_{1}$. Therefore, if we coordinatize $U$ as $U_{1} \oplus U_{2}$ where

$$
U_{1}=\left\{u \in U \mid B u \in V^{*}\left(Y_{1}\right) \text { and } D u \in Y_{1}\right\}
$$

and $U_{2}$ is an arbitrary complement of $U_{1}$, then equations (2.18)-(2.20) can be written in the form

$$
\begin{aligned}
\dot{x}_{1} & =(A+B F)_{11} x_{1}+B_{11} u_{1}, \\
y & =\left(C_{1}+D_{2} F_{21}\right) x_{1}+D_{1} u_{1}, \\
u_{2} & =F_{21} x_{1},
\end{aligned}
$$


where:

(i) $F$ satisfies (2.22) and (2.23), and

$$
\operatorname{im} F \subset U_{2}
$$

(ii) $F_{21}$ is the restriction of $F$ to $V^{*}\left(Y_{1}\right)$ considered as a mapping into $U_{2}$;

(iii) $(A+B F)_{11}$ is the restriction of $A+B F$ to $V^{*}\left(Y_{1}\right)$;

(iv) $B_{11}$ is the restriction of $B$ to $U_{1}$ taken as a mapping into $V^{*}\left(Y_{1}\right)$;

(v) $C_{1}$ is the restriction of $C$ to $V^{*}\left(Y_{1}\right)$.

So, we can summarize our conclusions as follows.

Theorem 2.1. Consider the system (2.1)-(2.3). Let $V^{*}\left(Y_{1}\right)$ be the limit of the sequence (2.4)-(2.5), and let $U_{1}$ be defined by (2.24). For any complement $U_{2}$ of $U_{1}$ in $U$, there exists a mapping $F: X \rightarrow U$ with im $F \subset U_{2}$ that satisfies (2.22) and (2.23), and the associated system (2.25)-(2.27) is externally equivalent to (2.1)-(2.3). Moreover, the action of $F$ on $V^{*}\left(Y_{1}\right)$ (and hence, the system (2.25)-(2.27)) is determined uniquely by the requirements (2.22), (2.23), and im $F \subset U_{2}$.

Proof. It remains to prove the uniqueness claim. Let $F$ and $F^{\prime}$ both satisfy the conditions (2.22), (2.23), and (2.28). Take $x \in V^{*}\left(Y_{1}\right)$, and write $F x=u, F^{\prime} x=u^{\prime}$. It follows from (2.22) and (2.23) that $B\left(u-u^{\prime}\right) \in V^{*}\left(Y_{1}\right)$ and $D\left(u-u^{\prime}\right) \in Y_{1}$, so that $u-u^{\prime} \in U_{1}$ by the definition of $U_{1}$. However, from (2.28) we also see that $u-u^{\prime} \in$ $U_{2}$. Because $U_{1} \cap U_{2}=\{0\}$, it follows that $u=u^{\prime}$, and the claim is proved.

Corollary 2.2. In the situation of the theorem, the reduction of the number of inputs that results from imposing the constraint (2.3) is equal to

$$
m_{\text {red }}=\operatorname{codim} Y_{1}-\operatorname{codim}\left(Y_{1}+\operatorname{im} G(s)\right) .
$$

Proof. The theorem that we just proved shows that the number of inputs in the constrained system is equal to

$$
m_{\mathrm{c}}=\operatorname{dim}\left\{u \in U \mid B u \in V^{*}\left(Y_{1}\right) \text { and } D u \in Y_{1}\right\} .
$$

Let $H$ denote any mapping such that ker $H=Y_{1}$; then (1.17) shows that the above quantity is equal to the dimension of the kernel of the rational mapping $H G(s)$. Denoting the original number of inputs by $m=\operatorname{dim} U$, we can write

$$
\begin{aligned}
m_{\mathrm{red}} & =m-m_{\mathrm{c}}=\operatorname{dim} U-\operatorname{dim} \operatorname{ker} H G(s)=\operatorname{dim} \operatorname{im} H G(s) \\
& =\operatorname{dim} \operatorname{im} G(s)-\operatorname{dim}[\operatorname{im} G(s) \cap \operatorname{ker} H] \\
& =\operatorname{dim}\left[\operatorname{im} G(s)+Y_{1}\right]-\operatorname{dim} Y_{1},
\end{aligned}
$$

which is equivalent to (2.29).

Remark 2.3. The number of independent constraints is given by codim $Y_{1}$. So, the corollary states in particular that the number of inputs can never be reduced by an amount larger than the number of independent constraints. 
It is seen that the constraints will not reduce the number of inputs if and only if the transfer matrix $G(s)$ maps into the constraint subspace $Y_{1}$. In general, we can say that only constraints on outputs that depend on the controllable part of the system will reduce the number of inputs.

An important thing to note in the theorem is that, once a choice has been made for a complement of $U_{1}=\left\{u \in U \mid B u \in V^{*}\left(Y_{1}\right)\right.$ and $\left.D u \in Y_{1}\right\}$ in $U$, the minimal state representation of (2.1)-(2.3) is essentially unique. So, the ambiguity of choosing a state representation for a system under output constraints is parametrized by the freedom we have in selecting a complement to a given subspace of the input space.

\section{Hamiltonian Systems}

We use the following definition of a linear Hamiltonian system in state space form, which is easily seen to be compatible with the definition given on pp. 111 and 150 of [S1].

Definition 3.1. Consider a linear system in input/state/output form

$$
\begin{aligned}
& \dot{x}(t)=A x(t)+B u(t), \\
& y(t)=C x(t) .
\end{aligned}
$$

Assume that the state space $X$ is equipped with a symplectic form denoted by $(\cdot, \cdot)$, and that the input and output spaces $U$ and $Y$ are dual with respect to a duality denoted by $\langle\cdot, \cdot\rangle$. The system (3.1)-(3.2) is said to be Hamiltonian if the following conditions are satisfied:

(i) $\left(A x_{1}, x_{2}\right)$ defines a symmetric form on $X$;

(ii) $(x, B u)=\langle C x, u\rangle$ for all $x \in X$ and $u \in U$.

We also assume that $B$ is injective.

The assumption on the injectivity of the input mapping $B$ helps to avoid some uninteresting singularities. Note that this assumption, under condition (ii), also implies that the output mapping $C$ is surjective: $u \in \operatorname{ker} B$ is equivalent to $0=$ $(x, B u)=\langle C x, u\rangle$ for all $x$, i.e., $u \in \operatorname{ker} B$ if and only if $u \in(\operatorname{im} C)^{\perp}$.

For a Hamiltonian system (3.1)-(3.2), the quadratic form $H(x)=\frac{1}{2}(A x, x)$ is called the Hamiltonian of the system, or the energy. The system is said to be time-reversible if $C A^{2 k} B=0$ for all nonnegative integers $k$ (see p. 200 of [S1] for a motivation of the terminology).

We now want to add the output constraint

$$
y(t) \in Y_{1} \quad \text { for all } t .
$$

To apply the general result, Theorem 2.1 , we have to compute $V^{*}\left(Y_{1}\right)$ and select a complement $U_{2}$ to the subspace $U_{1}$ defined by (2.24). In the general situation, there are no special candidates for the role of $U_{2}$, and all complements of $U_{1}$ are equally qualified. In the case of a Hamiltonian system, however, there is one particular candidate, namely the subspace $Y_{1}^{\perp}$ perpendicular to the constraint subspace $Y_{1}$. 
Under suitable circumstances, this is indeed a complement of $U_{1}$; one possible set of sufficient conditions is given in the lemma below.

Lemma 3.2. Let (3.1)-(3.2) be a Hamiltonian system, and assume it has uniformly second-order zeros at infinity (i.e., $C B=0$ and $C A B$ is invertible). Let a subspace $Y_{1}$ of $Y$ be given. Under these conditions, the subspace $V^{*}\left(Y_{1}\right)$ is given by

$$
V^{*}\left(Y_{1}\right)=\left\{x \in X \mid C x \in Y_{1} \text { and } C A x \in Y_{1}\right\},
$$

and $Y_{1}^{\perp}$ is complementary to the subspace $U_{1}=\left\{u \in U \mid B u \in V^{*}\left(Y_{1}\right)\right\}$ if and only if

$$
C A B Y_{1}^{\perp} \cap Y_{1}=\{0\} \text {. }
$$

Proof. Following the defining algorithm (2.4)-(2.5), we get (because $C B=0$ )

$$
\begin{aligned}
& V^{1}\left(Y_{1}\right)=\left\{x \in X \mid C x \in Y_{1}\right\}, \\
& V^{2}\left(Y_{1}\right)=\left\{x \in X \mid C x \in Y_{1} \text { and } C A x \in Y_{1}\right\}, \\
& V^{3}\left(Y_{1}\right)=\left\{x \in V^{2}\left(Y_{1}\right) \mid \exists u \in U \text { such that } C A^{2} x+C A B u \in Y_{1}\right\} .
\end{aligned}
$$

Because $C A B$ is invertible, we have $V^{3}\left(Y_{1}\right)=V^{2}\left(Y_{1}\right)$, so that $V^{*}\left(Y_{1}\right)=V^{2}\left(Y_{1}\right)$ is given by (3.7). Therefore, again using $C B=0$, we obtain

$$
U_{1}=\left\{u \in U \mid C A B u \in Y_{1}\right\}=(C A B)^{-1} Y_{1}
$$

which shows that the dimensions of $U_{1}$ and $Y_{1}$ are equal. So, $Y_{1}^{\perp}$ is a complement to $U_{1}$ if and only if $Y_{1}^{\perp} \cap U_{1}=\{0\}$; by (3.9), this is equivalent to (3.5).

Remark 3.3. It should be noted that (3.5) will hold for every subspace $Y_{1}$ of $Y$ if the matrix $C A B$ is positive definite. This is easy to see; suppose $u \in Y_{1}^{\perp}$ is such that $C A B u \in Y_{1}$, then we will have

$$
\langle C A B u, u\rangle=0 .
$$

If $C A B$ is positive definite, this implies that $u=0$. The positive definiteness condition can be interpreted in physical terms. If $u(t)$ has the dimension of a force and $y(t)$ that of a displacement, then $(C A B)^{-1}$ will have the dimension of a mass. Under the conditions of the theorem (uniformly second-order zeros at infinity), the matrix $(C A B)^{-1}$ is the leading term in the power series development around infinity of the inverse transfer function:

$$
G^{-1}(s)=(C A B)^{-1} s^{2}+\text { lower-order terms in } s .
$$

In a simple mechanical vibratory system of the form $M \ddot{y}+K y=u$, the inverse transfer function (also called "mechanical impedance," see for instance p. 119 of [D]) is obviously given by

$$
G^{-1}(s)=M s^{2}+K
$$

and the mass matrix $M$ might be determined from the impedance by multiplying by $s^{-2}$ and taking the limit as $s$ goes to infinity. Following the same procedure for (3.11) would give $(C A B)^{-1}$ as a result, and so we call this matrix the effective mass matrix of the system (3.1)-(3.2). The requirement that $C A B$ is positive definite can 
now be formulated as: the effective mass matrix of (3.1)-(3.2) should be positive definite.

If we let $C_{11}$ denote the restriction of $C$ to $V^{*}\left(Y_{1}\right)$ taken as a mapping into $Y_{1}$, and if we coordinatize $Y$ as $Y_{1} \oplus Y_{2}$ where $Y_{2}$ is an arbitrary complement to $Y_{1}$, then it already follows from Theorem 2.1 that an equivalent state space form for equations (3.1) and (3.2) under the constraint (3.3), with state space $X_{1}=V^{*}\left(Y_{1}\right)$, is given by

$$
\begin{aligned}
& \dot{x}_{1}=(A+B F)_{11} x_{1}+B_{11} u_{1}, \\
& y_{1}=C_{11} x, \\
& u_{2}=F_{21} x_{1}, \\
& y_{2}=0
\end{aligned}
$$

where $F$ is any mapping satisfying

$$
(A+B F) V^{*}\left(Y_{1}\right) \subset V^{*}\left(Y_{1}\right) .
$$

The notation here is as in the previous section; note that the requirement (2.23) is automatically satisfied in cases, such as the one considered in this section, where the mapping $D$ is equal to zero. The following theorem, which is the main result of this section, states what more can be said when $F$ is chosen such that its range space lies in the particular subspace $Y_{1}^{\perp}$. Similar results were given in [S3] (for a much more general situation than considered here) and in [H2], but in these it was not proved that these equations do indeed give an equivalent representation of the original system with constraints.

Theorem 3.4. Let (3.1)-(3.2) be a Hamiltonian system with uniformly second-order zeros at infinity. Let $Y_{1}$ be a subspace of $Y$, and consider the system (3.1)-(3.2) with the constraint (3.3). If the complementarity condition (3.5) is satisfied, a feedback mapping ranging in $Y_{1}^{\perp}$ may be selected that satisfies (3.17). The state space of the equivalent description (3.13)-(3.16) is then a symplectic space with respect to the form it inherits from the original state space $X$, and (3.13)-(3.14) is a Hamiltonian system with respect to this symplectic form and the induced duality between $Y_{1}$ and $U_{1}=$ $\left\{u \in U \mid B u \in V^{*}\left(Y_{1}\right)\right\}$. Finally, the energy function of (3.13)-(3.14) is the energy function of (3.1)-(3.2) restricted to $X_{1}$.

Proof. To show that $X_{1}=V^{*}\left(Y_{1}\right)$ inherits a symplectic structure from $X$, we use Lemma 1.1 with $\left\{x \mid C x \in Y_{1}\right\}$ in the role of the subspace $V$ and $V^{*}\left(Y_{1}\right)$ in the role of the subspace $W$. First, we note that the symplectic orthoplement of $\left\{x \mid C x \in Y_{1}\right\}$ is equal to $B Y_{1}^{\perp}$, because

$$
\begin{aligned}
\left\{x \mid C x \in Y_{1}\right\} & =\left\{x \mid\langle C x, u\rangle=0 \text { for all } u \in Y_{1}^{\perp}\right\} \\
& =\left\{x \mid(x, B u)=0 \text { for all } u \in Y_{1}^{\perp}\right\}=\left(B Y_{1}^{\perp}\right)^{(\perp)} .
\end{aligned}
$$

It follows from $C B=0$ that $B Y_{1}^{\perp}$ is contained in $\left\{x \mid C x \in Y_{1}\right\}$, so that, obviously,

$$
\left\{x \mid C x \in Y_{1}\right\} \cap\left\{x \mid C x \in Y_{1}\right\}^{(\perp)}=B Y_{1}^{\perp} .
$$


Therefore, Lemma 1.1 will give us the result we want if we can show that $V^{*}\left(Y_{1}\right)$ is a complement of $B Y_{1}^{\perp}$ in $\left\{x \mid C x \in Y_{1}\right\}$. To show that the two subspaces intersect only in 0, assume that $u \in Y_{1}^{\perp}$ is such that $B u \in V^{*}\left(Y_{1}\right)$. It follows from (3.7) that $C A B u \in C A B Y_{1}^{\perp} \cap Y_{1}=\{0\}$. Because $C A B$ is invertible, this indeed proves that $u=0$. To complete this part of the proof, we have to show that $\left\{x \mid C x \in Y_{1}\right\}=$ $V^{*}\left(Y_{1}\right)+B Y_{1}^{\perp}$. Take $x$ such that $C x \in Y_{1}$; we have to find $u \in Y_{1}^{\perp}$ such that $x-B u \in V^{*}\left(Y_{1}\right)$. Because $C A B Y_{1}^{\perp}$ is a complement of $Y_{1}$ in the output space $Y$, there exists a $u \in Y_{1}^{\perp}$ such that

$$
C A x-C A B u \in Y_{1} .
$$

Because we also have $C(x-B u)=C x \in Y_{1}$, we see that $x-B u \in V^{2}\left(Y_{1}\right)=V^{*}\left(Y_{1}\right)$, as desired.

Now, since $F$ maps into $Y_{1}^{\perp}$ and $B Y_{1}^{\perp}$ is contained in the symplectic orthoplement of $V^{*}\left(Y_{1}\right)$, we have

$$
\left((A+B F) x_{1}, x_{2}\right)=\left(A x_{1}, x_{2}\right)
$$

for $x_{1}$ and $x_{2}$ from $V^{*}\left(Y_{1}\right)$. It follows that condition (i) in the definition of a Hamiltonian system is satisfied, and that the Hamiltonian of the constrained system is the restriction to $V^{*}\left(Y_{1}\right)$ of the Hamiltonian of the original system. Because $U_{1}$ is a complement to $U_{2}=Y_{1}^{\perp}$, the duality between $U$ and $Y$ can be restricted to a duality between $U_{1}$ and $Y_{1}$. It follows immediately that condition (ii) in the definition of a Hamiltonian system is also satisfied by the constrained system.

Remark 3.5. Suppose that the dimension of the original state space is $2 n$, and that we impose $k$ constraints; i.e., the codimension of $Y_{1}$ is $k$. Under the conditions of the theorem, we know that the state space of the constrained system, $V^{*}\left(Y_{1}\right)$, is a complement to $B Y_{1}^{\perp}$ in $\left\{x \mid C x \in Y_{1}\right\}$. Because $B$ is injective and $C$ is surjective, it follows from this that the dimension of $V^{*}\left(Y_{1}\right)$ is $2 n-2 k$. This is the well-known property that "a system with $n$ degrees of freedom under $k$ constraints becomes a system with $n-k$ degrees of freedom." We have given here an algebraic proof of this fact; in textbooks, we usually find proofs that are based on some limit argument (see the classical reference [R1], but also the more recent treatment in [A2]).

We have seen that, under mild conditions, output constraints on a Hamiltonian system uniquely define a new Hamiltonian system. Some properties of the original system will go over to the constrained system; such properties are called "hereditary." In the following proposition, we list a number of hereditary properties.

Proposition 3.6. Under the conditions of Theorem 3.4, the constrained system (3.13)(3.14) will have uniformly second-order zeros at infinity. If the original system (3.1)(3.2) has a positive definite effective mass matrix, then the same will be true for the constrained system. If the original system is time-reversible, then so is the constrained system.

Proof. We use the notation of the theorem. Because $C B=0$, we have $C(A+B F) B=C A B$. This shows immediately that $C_{11}(A+B F)_{11} B_{11}$ is injective 
and hence invertible. The fact that $C B=0$ also implies that $C_{11} B_{11}=0$, and so we have shown that constrained system has uniformly second-order zeros at infinity.

Now, assume that the effective mass matrix of the original system, $(C A B)^{-1}$, is positive definite. Take $u$ from $U_{1}$. Using (3.21), we can write

$$
\left\langle C_{11}(A+B F)_{11} B_{11} u, u\right\rangle=((A+B F) B u, B u)=(A B u, u)=\langle C A B u, u\rangle \geq 0,
$$

with equality if and only if $u=0$. We see that the matrix $\left(C_{11}\left(f^{i}+B F\right)_{11} B_{11}\right)^{-1}$ is also positive definite, which proves our second claim.

It remains to show that the property of time-reversibility is hereditary. We first show that the following property holds for all $k \geq 0$ :

$$
\left((A+B F)^{k} x_{1}, x_{2}\right)=\left(A^{k} x_{1}, x_{2}\right) \quad\left(x_{1}, x_{2} \in V^{*}\left(Y_{1}\right)\right) .
$$

This property is trivially true for $k=0$, and its validity for $k=1$ is asserted by (3.21). The general case is proved by induction: suppose that (3.23) holds for certain $k$, then, for $x_{1}$ and $x_{2}$ from $V^{*}\left(Y_{1}\right)$,

$$
\begin{aligned}
\left((A+B F)^{k+1} x_{1}, x_{2}\right) & =\left(A(A+B F)^{k} x_{1}, x_{2}\right)=\left(A x_{2},(A+B F)^{k} x_{1}\right) \\
& =-\left((A+B F)^{k} x_{1}, A x_{2}\right)=-\left(A^{k} x_{1}, A x_{2}\right)=\left(A x_{2}, A^{k} x_{1}\right) \\
& =\left(A^{k+1} x_{1}, x_{2}\right) .
\end{aligned}
$$

In this derivation we used the validity of the formula for $k=1$, condition (i) in the definition of a Hamiltonian system, the sympletic property, the induction assumption, the symplectic property again, and condition (i) again. Now, suppose that the original system is time-reversible, i.e., the mappings appearing in (3.1)-(3.2) satisfy $C A^{2 k} B=0$ for all $k \geq 0$. By condition (ii) of the definition and the property that we just proved, it then follows that

$$
\left\langle C(A+B F)^{2 k} B u_{1}, u_{2}\right\rangle=0
$$

for all $u_{1}$ and $u_{2}$ from $U_{1}$, which, by the fact that $U_{1}$ and $Y_{1}$ are dual spaces, is enough to show that $C_{11}\left((A+B F)_{11}\right)^{2 k} B_{11}=0$ for all $k \geq 0$.

This proposition allows us to conclude, for instance, that any system that is obtained by putting linear constraints on the outputs in the Hamiltonian system $M \ddot{y}+K y=$ $u$ ( $M$ and $K$ symmetric, $M$ positive definite) is a time-reversible Hamiltonian system with a positive definite effective mass matrix.

\section{Gradient Systems}

The following definition of a linear gradient system in input/state/output form is used here; it is easily seen to be equivalent to the one given on p. 224 of [S1].

Definition 4.1. Consider a linear system in input/state/output form (3.1)-(3.2). Assume that the state space $X$ is equipped with a nondegenerate quadratic form denoted by $[\cdot, \cdot]$, and that the input and output spaces are dual with respect to a duality denoted by $\langle\cdot, \cdot\rangle$. The system (3.1)-(3.2) is said to be a gradient system (with 
respect to the quadratic form $[\cdot, \cdot]$ and the duality $\langle\cdot, \cdot\rangle)$ if the following requirements hold:

(i) $A$ is symmetric with respect to $[\cdot, \cdot]$;

(ii) $[B u, x]=\langle u, C x\rangle$ for all $u \in U$ and $x \in X$.

We also assume that $B$ is injective.

As in the case of Hamiltonian systems, injectivity of $B$ implies surjectivity of $C$. We call the quadratic form $\frac{1}{2}[A x, x]$ the generalized potential of the system; note that $A x$ may be seen as the gradient of $\frac{1}{2}[A x, x]$ with respect to the symmetric form $[\cdot, \cdot]$.

We now consider the constraint (3.3) in this context. As before, we are looking for conditions under which we can define a constrained system which inherits, in a natural way, the special structure of the original system. We define $U_{1}=$ $\left\{u \mid B u \in V^{*}\left(Y_{1}\right)\right\}$ as in the Hamiltonian case, and look for complements of $U_{1}$ in $U$. Again, a natural candidate is $Y_{1}^{\perp}$. It turns out that, as soon as this candidate qualifies, the description that we derive from it has all the desired properties.

Theorem 4.2. Let (3.1)-(3.2) be a gradient system, considered under the constraint (3.3), and assume that $Y_{1}^{\perp}$ is a complement to $U_{1}=\left\{u \mid B u \in V^{*}\left(Y_{1}\right)\right\}$ in $U$. Let $F: X \rightarrow U$ be a feedback mapping such that im $F \subset Y_{1}^{\perp}$ and such that $V^{*}\left(Y_{1}\right)$ is $(A+B F)$-invariant. The equivalent state space representation (3.13)-(3.16) is then such that (3.13)-(3.14) is a gradient system with respect to the restriction of the form $[\cdot, \cdot]$ to $V^{*}\left(Y_{1}\right)$ and of the duality $\langle\cdot, \cdot\rangle$ to the pair of spaces $\left(U_{1}, Y_{1}\right)$. The generalized potential function of this system coincides with the generalized potential of the original system restricted to $V^{*}\left(Y_{1}\right)$.

Proof. The fact that (3.13)-(3.16) is a representation of the system (3.1)-(3.2) under the constraint (3.3) follows from the general theory. Because $F$ maps into $Y_{1}^{\perp}$ and $V^{*}\left(Y_{1}\right)$ is mapped by $C$ into $Y_{1}$, we have

$$
\left[(A+B F) x_{1}, x_{2}\right]=\left[A x_{1}, x_{2}\right]+\left\langle F x_{1}, C x_{2}\right\rangle=\left[A x_{1}, x_{2}\right]
$$

for all $x_{1}$ and $x_{2}$ from $V^{*}\left(Y_{1}\right)$. This shows that condition (i) of the definition of a gradient system is satisfied, and also that the generalized potentials of the unconstrained system and the constrained system are equal on $V^{*}\left(Y_{1}\right)$. The fact that the duality between $U$ and $Y$ can be restricted to $U_{1}$ and $Y_{1}$ follows from the assumption that $U_{1}$ is complementary to $Y_{1}^{\perp}$, and condition (ii) for the constrained system is then immediate from the corresponding property of the original system.

If the symmetric form on $X$ is definite (i.e., $[x, x]=0$ implies $x=0$ so the form is either positive or negative definite), then we can show that the complementarity condition is satisfied for all possible restrictions.

Proposition 4.3. Assume that the system (3.1)-(3.2) is a gradient system with respect to a nondegenerate symmetric form $[\cdot, \cdot]$ in the state space $X$ and a duality $\langle\cdot, \cdot\rangle$ between $U$ and $Y$. Assume that the symmetric form $\left[\cdot^{\cdot} \cdot\right]$ is definite. Under these 
conditions, the subspace $U_{1}=\left\{u \in U \mid B u \in V^{*}\left(Y_{1}\right)\right\}$ is complementary to $Y_{1}^{\perp}$ for any subspace $Y_{1}$ of $Y$.

Proof. We first show that the definiteness of the symmetric form on $X$ implies that $C B$ is invertible. Because $C B$ is square, it is sufficient to prove the injectivity. So, suppose that $C B u=0$; then

$$
[B u, B u]=\langle C B u, u\rangle=0
$$

which implies that $u=0$. From the invertibility of $C B$, it follows that

$$
V^{*}\left(Y_{1}\right)=\left\{x \in X \mid C x \in Y_{1}\right\} .
$$

As a consequence, we have

$$
U_{1}=\left\{u \in U \mid C B u \in Y_{1}\right\}=(C B)^{-1} Y_{1} .
$$

This shows that $\operatorname{dim} U_{1}+\operatorname{dim} Y_{1}^{\perp}=\operatorname{dim} U$, so that the complementarity condition will hold if $U_{1} \cap Y_{1}^{\perp}=\{0\}$.

For gradient systems in general, we have

$$
\left\{x \mid C x \in Y_{1}\right\}=\left(B Y_{1}\right)^{[\perp]}
$$

(proof as in the Hamiltonian case, see (3.18)). When the form is definite, this implies that $V^{*}\left(Y_{1}\right)$ has zero intersection with $B Y_{1}^{\perp}$. Now, take $u \in U_{1} \cap Y_{1}^{\perp}$; then $B u \in$ $V^{*}\left(Y_{1}\right) \cap Y_{1}^{\perp}=\{0\}$, so that $u=0$.

In gradient systems that arise as descriptions of RLC networks, the form on the state space $X$ is usually not definite, unless we have either no capacitors or no inductors in the network. In order to obtain suitable sufficient conditions for output constraints to be well behaved in the context of general RLC networks, a further analysis of the gradient systems defined by such networks should be undertaken (following up on the work in [BM]); however, we will not do this here.

The invertibility of the mapping $C B$, which, as we have seen, holds automatically when the form on $X$ is definite, is in itself already enough to obtain the expression (4.3) for $V^{*}\left(Y_{1}\right)$. This allows us to draw the conclusion that a gradient system with uniformly first-order zeros at infinity and with state space dimension $n$ (" $n$ degrees of freedom") becomes a system with $n-k$ degrees of freedom if $k$ linear constraints are imposed on the outputs. Note that the property of having uniformly first-order zeros at infinity is "hereditary": if the original system (3.1)-(3.2) has this property, then the same holds for the constrained system (3.13)-(3.14): $C$ maps $V^{*}\left(Y_{1}\right)$ into $Y_{1}$, and this implies that if $C_{11} B_{11} u=0$, then $C B u=0$, so that $C_{11} B_{11}$ will be invertible if $C B$ is. (We also use here the fact that the invertibility of $C B$ implies that $C_{11} B_{11}$ is square.)

\section{Examples}

We work out two simple examples in order to illustrate the abstract theory and to show that the theory leads to the answers that we should expect. 


\section{Mechanical Example}

Our first example is the same as in Section 1. State space equations for a mass on an ideal spring can be written as follows:

$$
\begin{aligned}
\frac{d}{d t}\left(\begin{array}{l}
q \\
p
\end{array}\right)(t) & =\left(\begin{array}{cc}
0 & m^{-1} \\
-k & 0
\end{array}\right)\left(\begin{array}{l}
q \\
p
\end{array}\right)(t)+\left(\begin{array}{l}
0 \\
1
\end{array}\right) u(t), \\
y(t) & =\left[\begin{array}{ll}
1 & 0
\end{array}\right]\left(\begin{array}{l}
q \\
p
\end{array}\right)(t) .
\end{aligned}
$$

The force (input) is $u(t)$, the displacement (output) is $y(t)$. It is easily verified that the above equations constitute a Hamiltonian system with respect to the symplectic form

$$
\left(\left(\begin{array}{l}
p \\
q
\end{array}\right),\left(\begin{array}{l}
\tilde{p} \\
\tilde{q}
\end{array}\right)\right)=q \tilde{p}-\tilde{q} p
$$

on $X=\mathbb{R}^{2}$ and the duality

$$
\langle u, y\rangle=u y
$$

between $U=\mathbb{R}$ and $Y=\mathbb{R}$. The system has a second-order zero at infinity. Let us now take two such systems and connect them by requiring that the outputs (displacements) must be the same, i.e., the two masses are firmly attached to each other. The system before connection is described by the matrices

$$
A=\left(\begin{array}{cccc}
0 & m_{1}^{-1} & 0 & 0 \\
-k_{1} & 0 & 0 & 0 \\
0 & 0 & 0 & m_{2}^{-1} \\
0 & 0 & -k_{2} & 0
\end{array}\right), \quad B=\left(\begin{array}{ll}
0 & 0 \\
1 & 0 \\
0 & 0 \\
0 & 1
\end{array}\right), \quad C=\left(\begin{array}{cccc}
1 & 0 & 0 & 0 \\
0 & 0 & 1 & 0
\end{array}\right)
$$

and is a Hamiltonian system having uniformly second-order zeros at infinity with respect to the symplectic form

$$
\left(\left(\begin{array}{c}
q_{1} \\
p_{1} \\
q_{2} \\
p_{2}
\end{array}\right),\left(\begin{array}{c}
\tilde{q}_{1} \\
\tilde{p}_{1} \\
\tilde{q}_{2} \\
\tilde{p}_{2}
\end{array}\right)\right)=q_{1} \tilde{p}_{1}-\tilde{q}_{1} p_{1}+q_{2} \tilde{p}_{2}-\tilde{q}_{2} p_{2}
$$

on $X=\mathbb{R}^{4}$ and the duality

$$
\left\langle\left(\begin{array}{l}
y_{1} \\
y_{2}
\end{array}\right),\left(\begin{array}{l}
u_{1} \\
u_{2}
\end{array}\right)\right\rangle=y_{1} u_{1}+y_{2} u_{2}
$$

between $Y=\mathbb{R}^{2}$ and $U=\mathbb{R}^{2}$. The connection constraint is expressed by

$$
y(t) \in Y_{1}=\operatorname{span}\left\{\left(\begin{array}{l}
1 \\
1
\end{array}\right)\right\}
$$


We readily compute that a basis for $V^{*}\left(Y_{1}\right)$ is given by the two vectors

We also have

$$
\left(\begin{array}{l}
1 \\
0 \\
1 \\
0
\end{array}\right), \quad\left(\begin{array}{c}
0 \\
m_{1} \\
0 \\
m_{2}
\end{array}\right)
$$

$$
Y_{1}^{\perp}=\operatorname{span}\left\{\left(\begin{array}{c}
1 \\
-1
\end{array}\right)\right\}
$$

and so we are looking for a mapping $F: X \rightarrow U$ which ranges in this subspace and which is such that $V^{*}\left(Y_{1}\right)$ becomes $A+B F$-invariant. Upon computing, we find that this requires

$$
F\left(\begin{array}{cc}
1 & 0 \\
0 & m_{1} \\
1 & 0 \\
0 & m_{2}
\end{array}\right)=\left(\begin{array}{c}
1 \\
-1
\end{array}\right)\left[\frac{m_{2} k_{1}-m_{1} k_{2}}{m_{1}+m_{2}} \quad 0\right] .
$$

Here, we need that $m_{1}+m_{2}$ is not equal to zero; note that this is precisely the condition for $Y_{1}^{\perp}$ to be complementary to $U_{1}$. We assume that this condition is fulfilled. It then follows from (5.11) that the action of $A+B F$ on $V^{*}\left(Y_{1}\right)$ is given by

$$
(A+B F)\left(\begin{array}{cc}
1 & 0 \\
0 & m_{1} \\
1 & 0 \\
0 & m_{2}
\end{array}\right)=\left(\begin{array}{cc}
1 & 0 \\
0 & m_{1} \\
1 & 0 \\
0 & m_{2}
\end{array}\right)\left(\begin{array}{cc}
0 & 1 \\
-\frac{k_{1}+k_{2}}{m_{1}+m_{2}} & 0
\end{array}\right) .
$$

In order to obtain a matrix representation for the connected system, we have to select basis vectors in $V^{*}\left(Y_{1}\right), U_{1}$, and $Y_{1}$. It is not difficult to show (see p. 200 of [S1]) that "canonical" bases may be chosen in the following way for every Hamiltonian system (3.1)-(3.2) satisfying $C B=0$. First select an arbitrary basis for the output space $Y$. Next, determine the dual basis $u_{1}, \ldots, u_{m}$ for the input space $U$. Finally, it is possible to find a symplectic basis $\left\{q_{1}, \ldots, q_{n}, p_{1}, \ldots, p_{n}\right\}$ for the state space $X$ in such a way that $B u_{i}=p_{n-m+i}$ for $i=1, \ldots, m$.

We can carry out this procedure in the case at hand. A basis vector for the output space $Y_{1}$ is $\left[\begin{array}{ll}1 & 1\end{array}\right]^{\mathrm{T}}$. The dual basis vector in $U_{1}$ is $\left(m_{1}+m_{2}\right)^{-1}\left[\begin{array}{ll}m_{1} & m_{2}\end{array}\right]^{\mathrm{T}}$. A corresponding symplectic basis for $X$ is given by

$$
\left\{\left(\begin{array}{l}
1 \\
0 \\
1 \\
0
\end{array}\right),\left(m_{1}+m_{2}\right)^{-1}\left(\begin{array}{c}
0 \\
m_{1} \\
0 \\
m_{2}
\end{array}\right)\right\} \text {. }
$$

The matrices describing the connected system are then

$$
(A+B F)_{11}=\left(\begin{array}{cc}
0 & \left(m_{1}+m_{2}\right)^{-1} \\
-\left(k_{1}+k_{2}\right) & 0
\end{array}\right), \quad B_{11}=\left(\begin{array}{l}
0 \\
1
\end{array}\right), \quad C_{11}=\left[\begin{array}{ll}
1 & 0
\end{array}\right] .
$$




\section{An Electrical Network}

For our second example let us consider the parallel connection of two capacitors. Before connection, the two capacitors can be described by the equations

$$
\begin{aligned}
\frac{d}{d t}\left(\begin{array}{l}
x_{1} \\
x_{2}
\end{array}\right)(t) & =0 \cdot\left(\begin{array}{l}
x_{1} \\
x_{2}
\end{array}\right)(t)+\left(\begin{array}{ll}
1 & 0 \\
0 & 1
\end{array}\right)\left(\begin{array}{l}
u_{1} \\
u_{2}
\end{array}\right)(t), \\
\left(\begin{array}{l}
y_{1} \\
y_{2}
\end{array}\right)(t) & =\left(\begin{array}{cc}
C_{1}^{-1} & 0 \\
0 & C_{2}^{-1}
\end{array}\right)\left(\begin{array}{l}
x_{1} \\
x_{2}
\end{array}\right)(t),
\end{aligned}
$$

where the inputs are currents and the outputs are voltages, and $C_{1}$ and $C_{2}$ are the capacitances of the two capacitors. This system is a gradient system with respect to the quadratic form

$$
\left[\left(\begin{array}{l}
x_{1} \\
x_{2}
\end{array}\right),\left(\begin{array}{l}
x_{1} \\
x_{2}
\end{array}\right)\right]=C_{1}^{-1} x_{1}^{2}+C_{2}^{-1} x_{2}^{2}
$$

on $X$ and the duality

$$
\left\langle\left(\begin{array}{l}
y_{1} \\
y_{2}
\end{array}\right),\left(\begin{array}{l}
u_{1} \\
u_{2}
\end{array}\right)\right\rangle=y_{1} u_{1}+y_{2} u_{2}
$$

between $Y$ and $U$. Now, establishing a parallel connection between the two capacitors means that the voltages across the two capacitors must be equal, which leads to the output constraint

$$
y_{1}(t)=y_{2}(t)
$$

So the constraint subspace $Y_{1}$ is given by

$$
Y_{1}=\operatorname{span}\left\{\left(\begin{array}{l}
1 \\
1
\end{array}\right)\right\} \text {. }
$$

We easily compute that

$$
U_{1}=\operatorname{span}\left\{\left(\begin{array}{l}
C_{1} \\
C_{2}
\end{array}\right)\right\}
$$

so that $U_{1}$ is complementary to $Y_{1}^{\perp}=\operatorname{span}\left\{\left[\begin{array}{ll}1 & -1\end{array}\right]^{\mathrm{T}}\right\}$ if and only if $C_{1} \neq-C_{2}$. Assuming this, we have to find a feedback mapping $F$ ranging in $Y_{1}^{\perp}$ such that $V^{*}\left(Y_{1}\right)=\operatorname{span}\left\{\left[\begin{array}{ll}C_{1} & C_{2}\end{array}\right]^{\mathrm{T}}\right\}$ becomes $(A+B F)$-invariant. This is satisfied by taking $F=0$, which leads to $(A+B F)_{11}=0$. We can take $\left[\begin{array}{ll}1 & 1\end{array}\right]^{\mathrm{T}}$ as a basis vector for $Y_{1}$; the dual basis vector in $U_{1}$ is $\left(C_{1}+C_{2}\right)^{-1}\left[\begin{array}{ll}C_{1} & C_{2}\end{array}\right]^{\mathrm{T}}$. Taking (nominally) the same vector as a basis vector for $X_{1}$, we obtain $B_{11}=1$. Finally, $C_{11}$ is then given by $\left(C_{1}+C_{2}\right)^{-1}$, because

$$
\left(\begin{array}{cc}
C_{1}^{-1} & 0 \\
0 & C_{2}^{-1}
\end{array}\right) \frac{1}{C_{1}+C_{2}}\left(\begin{array}{l}
C_{1} \\
C_{2}
\end{array}\right)=\frac{1}{C_{1}+C_{2}}\left(\begin{array}{l}
1 \\
1
\end{array}\right)
$$

The equations for the connected system now take the form

$$
\begin{aligned}
& \dot{x}(t)=u(t), \\
& y(t)=\left(C_{1}+C_{2}\right)^{-1} x(t) .
\end{aligned}
$$


Here, the old inputs (currents) are expressed in terms of the new input by

$$
\left(\begin{array}{l}
u_{1} \\
u_{2}
\end{array}\right)=\frac{1}{C_{1}+C_{2}}\left(\begin{array}{l}
C_{1} \\
C_{2}
\end{array}\right) u
$$

so that the new input can be written in terms of the old inputs as

$$
u=u_{1}+u_{2} \text {. }
$$

\section{Conclusions}

It has been shown in this paper that the transformation of a linear with output constraints to standard input/state/output form leads to an essentially nonunique result, and that the indeterminacy can be described by the freedom we have in selecting a complement to a given subspace in the input space. For the more specific categories of linear Hamiltonian systems and linear gradient systems, the notion of energy, expressed through special vector space structures, serves to remove the indeterminacy, and leads to results that are familiar from physical modeling. We have thus shown how the general theory of transformations of linear systems can be connected to the standard methods of physical modeling, at least for the case of linear systems under output constraints.

The analysis has also revealed a "nondegeneracy" condition, which appears in geometric terms as the requirement that two given subspaces should be complementary. The viewpoint used in this paper seems less suitable for a treatment of the particular cases in which the complementarity condition does not hold. An alternative framework for analysis can be set up by prescribing new external variables in conjunction with the output constraints. This point of view is currently under investigation.

\section{References}

[AM] R. A. Abraham and J. E. Marsden, Foundations of Mechanics, 2nd edn., Addison-Wesley, Reading, MA, 1978.

[A1] B. D. O. Anderson, Output-nulling invariant and controllability subspaces, Proceedings of the Sixth World Congress of the International Federation of Automatic Control, Cambridge, MA, 1975, paper 43.6.

[A2] V. I. Arnold, Mathematical Methods of Classical Mechanics, Springer-Verlag, New York, 1978.

[BY] H. Blomberg and R. Ylinen, Algebraic Theory for Multivariable Linear Systems, Academic Press, London, 1983.

[BM] R. K. Brayton and J. K. Moser, A theory of nonlinear networks, Quart. Appl. Math., 22 (1964), 1-33 (Part I), 81-104 (Part II).

[CM] J. P. Corfmat and A. S. Morse, Control of linear systems through specified input channels, SIAM J. Control Optim., 14 (1976), 163-175.

[D] J. P. Den Hartog, Mechanical Vibrations, Dover, New York, 1985; reprint of original 4th edn., 1956.

[H1] M. L. J. Hautus, The formal Laplace transform for smooth linear systems, in Mathematical Systems Theory (E. G. Marchesini and S. K. Mitter, eds.), pp. 29-47, Lecture Notes in Economics and Mathematical Systems, Vol. 131, Springer-Verlag, New York, 1976.

[H2] J. Hoekstra, Hamiltonse systemen met beperkingen op de uitgang (Hamiltonian systems with 
out put restrictions), M.Sc. thesis, Department of Applied Mathematics, Twente University, 1988 (in Dutch).

[L1] S. Lang, Algebra, Addison-Wesley, Reading, MA, 1965.

[L2] D. G. Luenberger, Dynamic equations in descriptor form, IEEE Trans. Automat. Control, 22 (1977), 312-321.

[M1] C. C. MacDuffee, The Theory of Matrices, Chelsea, New York, 1956; reprint of original, 1933.

[M2] M. Malabre, Structure à l'infini des triplets invariants: Application à la poursuite parfaite de modèle, in Analysis and Optimization of Systems (A. Bensoussan and J. L. Lions, eds.), pp. 43-53, Lect ure Notes in Control and Information Sciences, Vol. 44, Springer-Verlag, Berlin, 1982.

[NS] H. Nijmeijer and J. M. Schumacher, On the inherent integration structure of nonlinear systems, IM A J. Math. Control Inform. 2 (1985), 87-107.

[R1] J. W. S. Rayleigh, The Theory of Sound, Vol. 1, Dover, New York, 1945; reprint of original, 1894.

[R2] H. H. Rosenbrock, State Space and Multivariable Theory, Wiley, New York, 1970.

[S1] A. J. van der Schaft, System Theoretic Descriptions of Physical Systems, CWI Tract 3, Centre for Mathematics and Computer Science, Amsterdam, 1984.

[S2] A. J. van der Schaft, On realization of nonlinear systems described by higher-order differential equations, Math. Systems Theory, 19 (1987), 239-275.

[S3] A. J. van der Schaft, Equations of motion for Hamiltonian systems with constraints, J. Phys. A, 20 (1987), 3271-3277.

[S4] J. M. Schumacher, Transformations of linear systems under external equivalence, Linear Algebra Appl., 102 (1988), 1-34.

[W1] J. C. Willems, Input-output and state space representations of finite-dimensional linear timeinvariant systems, Linear Algebra Appl., 50 (1983), 581-608.

[W2] J. C. Willems, From time series to linear system, Part I: Finite-dimensional linear time-invariant systems, Automatica, 22 (1986), 561-580.

[W3] W. M. Wonham, Linear Multivariable Control: a Geometric Approach, 2nd edn., Springer-Verlag, New York, 1979. 